\title{
Towards a Traceability System Based on RFID Technology to Check the Content of Pallets within Electronic Devices Supply Chain
}

\author{
Ignacio Angulo, ${ }^{1}$ Asier Perallos, ${ }^{1}$ Leire Azpilicueta, ${ }^{2}$ Francisco Falcone, ${ }^{1,2}$ \\ Unai Hernandez-Jayo, ${ }^{1}$ Asier Moreno, ${ }^{1}$ and Ignacio Julio García Zuazola ${ }^{1}$ \\ ${ }^{1}$ Deusto Institute of Technology (DeustoTech), University of Deusto, 48007 Bilbao, Spain \\ ${ }^{2}$ Electrical and Electronic Engineering Department, Public University of Navarra, 31006 Pamplona, Spain
}

Correspondence should be addressed to Francisco Falcone; francisco.falcone@unavarra.es

Received 15 April 2013; Revised 4 July 2013; Accepted 19 July 2013

Academic Editor: Mira Trebar

Copyright (c) 2013 Ignacio Angulo et al. This is an open access article distributed under the Creative Commons Attribution License, which permits unrestricted use, distribution, and reproduction in any medium, provided the original work is properly cited.

In the last few years there has been a growing interest in smart solutions capable of dealing with the traceability of products and materials to improve logistical processes. Most of the existing solutions have been designed without considering the difficulties of deploying traceability systems in the storehouses currently working, not dealing with specific needs, such as environment characteristics or time required to be handled by workers. In this paper, in order to test the viability of its application, a first prototype of a traceability system capable of checking the content of pallets loaded with electronic devices is presented. It is based on ultra-high frequency (UHF) radio frequency identification (RFID) technology using passive tags. A holistic approach has been adopted to design the system: it begins with a radioelectrical characterization of the environment where the check points will be implemented, continues with the integration of a set of data acquisition and wireless communication devices, and ends with a logistics information system able to provide final user services. The combination of physical layer analysis with a top layer system view can aid the planning as well as operational phase of this type of RFID system within a logistic chain.

\section{Introduction}

Assuring the traceability of goods and its veracity represents a key factor in the competitiveness of many industries in developed countries. First, it is a way to compete against lowcost products with less origin guarantee and processing information. Second, in those markets regulated by governments, where all competitors are offering the same products at similar prices and service quality is a decisive factor, traceability of products represents a key factor to its improvement.

In the last few years there has been a considerable interest in the global concept of traceability of products and materials. Traceability processes traditionally required manually checking each item of a product and taking notes of its production processes. Thus, there is a growing need for smart solutions in logistics to deal with this challenge and improve the supply chain. Several research works have been developed which explore different aspects of traceability of goods $[1,2]$ applied to different types of industries $[3,4]$ and needs [5]. Even more important is the interest in sectors where traceability is not only a productive benefit, but a health or safety necessity. This is the case of food or pharmaceutical ones, where recently some work related to traceability of medicines has been published [6].

The main problem is that most of these existing solutions have been designed without considering the difficulties of deploying traceability systems in the storehouses currently working. Thus, they are not capable of dealing with specific needs that exist in certain sectors, such as the nondesired intrusiveness with the workers or the time required to be handled. Radio technologies could be a solution to systematize the capture of traceability data, its management, and, importantly, its sharing amongst partners in a value chain. Wireless 
networks, such as $\mathrm{WiFi}$, or radiofrequency identification technologies, such as RFID, are some examples of possible radio technologies involved in improving traceability methods.

Radiofrequency identification (RFID) system is an autoidentification method that can read codes that were previously stored in small transponders/tags wirelessly. Unlike barcodes, RFID does not require imminent handling, no line of sight is required between the reader and the tags, and these ones provide greater storage (64 bits, 96 bits, and 496 bits). The tags can be active (battery powered) or passive. This technology uses attached tags for monitoring and identifying objects in an omnidirectional fashion, creating tremendous benefits in a very different kind of applications like traceability of goods, baggage management, livestock tracking, and supply chain management [7].

In this paper, in order to test its feasibility, a first prototype of a traceability system capable of checking the content of containers (pallet) loaded with electronic devices is explored. The system is based on a set of check points distributed along the agents of the supply chain which are able to identify every electronic device contained in each pallet. Thus, the system is capable of detecting, by comparison with previous readings, if some of them are lost during the distribution process, when, and the area where the theft or loss has occurred at the specific stage in the distribution chain. The identification is done by ultrahigh frequency (UHF) RFID technology using passive tags, adopting a nonintrusive approach. A logistics software system is responsible for gathering the data in each check point, which are sent using available WiFi networks, and detecting incidences.

The rest of the paper is organized as follows. Section 2 presents some problems that occurred during the distribution of electronic devices, the partners involved, and the specific scenario where the proposed traceability system would be deployed and finally gives an overview of the benefits that this system would generate. Section 3 includes a radioelectrical characterization of the environment where the check points will be implemented. Section 4 describes the current hardware and software tracking prototype implementation, considering the different parts which conform it: the RFID based check points, the logistics software system, and the set of data acquisition and wireless communication devices. And finally, conclusions and references end the paper.

\section{Problem Description}

Nowadays, when small or medium sized goods must be delivered in big quantities, pallets are the most used platform to bring them together. Goods can often be packaged individually or some of them are saved in the same cardboard box. Then, the boxes that must be delivered in the same destination are placed on a pallet and wound with plastic or another material (sometimes a wood protection box is used) with the aim of maintaining all the freight together during the transportation. In Europe, the maximum dimensions of the pallets are $1.8 \mathrm{~m}$ height and $1.2 \mathrm{~m}$ width.
Each element of the freight can be identified using different techniques, being barcodes the most common one. But this system is not useful if the pallet is already composed: the elements that are not in the edge of the pallet cannot be read because there is not a line of sight between the barcodes and the reader. Moreover, the reading of the barcodes is performed one by one, and this task takes a certain time. Another alternative is not to identify each element of the pallet but to assign one code to the whole pallet. In a database this code represents and relates all the elements of the pallet. The drawback of this technique is that in case one element is stolen or lost, this event is not detected until the pallet is unpacked and all the goods are checked.

This situation can be solved if all the elements are equipped with RFID tag and an RF reader is placed in the passing area of the pallet. With the RFID based system presented in this paper, all the elements of the pallet can be identified and registered in the traceability or stocking application (no line of sight is required to carry out the reading) at the same time slot. But in this scenario, the successfulness of the reading depends mainly on the propagation characteristics inside the pallet and the distance between the reader and the tags. The materials used to package the goods and their own physical composition determine the attenuation, absorption, and reflection of the RF signal detected by the RF reader once the RFID tag has been excited.

In the specific case of the consumer electronic market, large surfaces and group of stores concentrate mainly on the sales of these products. The products can be supplied from producers or from assemblers and are transported by logistics operators to selling points and coded and displayed on the shelves.

To make it easier and more competitive, large stores are pursuing initiatives to manage the traceability of high value goods. For example, as part of GS1 Italy's i.Trace initiative [8], MediaMarkt, a leading European electronics retailer, in partnership with DHL, Sony, and Samsung, recently completed a successful pilot of EPC-enabled RFID technology for highvalue consumer electronics. In this pilot 14,000 units were tagged, including notebooks, digital cameras, video games, and mobile phones. A lift truck driver transports completed pallets through a dock door portal equipped with an RFID reader. By moving through the portal, the system automatically reads the tags and records the quantities and serial numbers of each stock-keeping unit (SKU) that is being loaded into the truck. At the store's receiving dock, inbound pallets are again transported through another gateway portal and are automatically received. A second gateway portal between the store's backroom and the sales floor automatically traces and records the individual serial numbers that are being moved to the store floor.

In the case of purchasing centers, the diversity of shops grouped further complicates the development of a solution that fits all. Sometimes the small size of the storage infrastructure complicates the installation of infrastructures so that, in the proposed system, the identification of the goods at the point of sale is carried out using a low-cost portable reader. 
In this common scenario, the presented solution will be carried out in three phases.

(1) The main warehouse of the purchasing center will be provided with an UHF RFID portal in order to detect and validate the SKU of the devices sent by producers through their own logistics operator.

(2) The warehouse staff will validate each pallet or package shipped to stores through its own shuttle service using a handheld reader, deployed on every delivery vehicle.

(3) Inventory managers at the stores will validate the receipt of the requested units in each purchase order using the same reader related to the previous stage.

This solution also enhances the security of the supply chain leading to fewer losses, reduces the time it takes to process store shipments, and provides inventory managers with a method to detect "stock-out" events.

\section{Radioelectrical Analysis}

Channel performance directly determines the quality of the communication in terms of sensitivity, capacity, and latency. Therefore, a very clear understanding of the channel must be pursued to get high-quality and high-capacity transmission of the useful information to give an efficient service. In order to analyze the impact of an RFID system on a logistics company, a deterministic method based on an in-house developed 3D ray launching code $[6,9-12]$, tested in complex indoor environments, is used. The assessment on electromagnetic spectrum is of importance in order to model overall performance of the system under analysis in terms of coverage and capacity analysis. A 3D ray launching algorithm has been implemented in-house based on Matlab programming environment. The algorithm is based on geometrical optics (GO) and geometrical theory of diffraction (GTD). The rays that are considered in GO are only direct, reflected, and refracted rays. Abrupt transition areas may occur because of this, corresponding to the boundaries of the region where these rays exist. To complement the GO theory, the diffracted rays are introduced with the GTD and its uniform extension, the uniform GTD (UTD). The purpose of these rays is to remove the field discontinuities and to introduce proper field corrections, especially in the zero-field regions predicted by GO.

Within the considered scenario, several sources (which can emulate the transmitter antennas of the readers) can be placed, in which wireless power is converted into a finite number of rays launched within a solid angle. Parameters such as frequency of operation, radiation diagram of the antennas, number of reflections, separation angle between rays, and cuboids dimension can be fixed. The commitment between accuracy and computational time is acquired with the number of launching rays and the cuboids size of the considered scenario. The benefit of this approach in radioelectric estimation is the fact that the proposed 3D ray launching algorithm can be employed in order to compute RFID reader link balance within the completed indoor scenario,

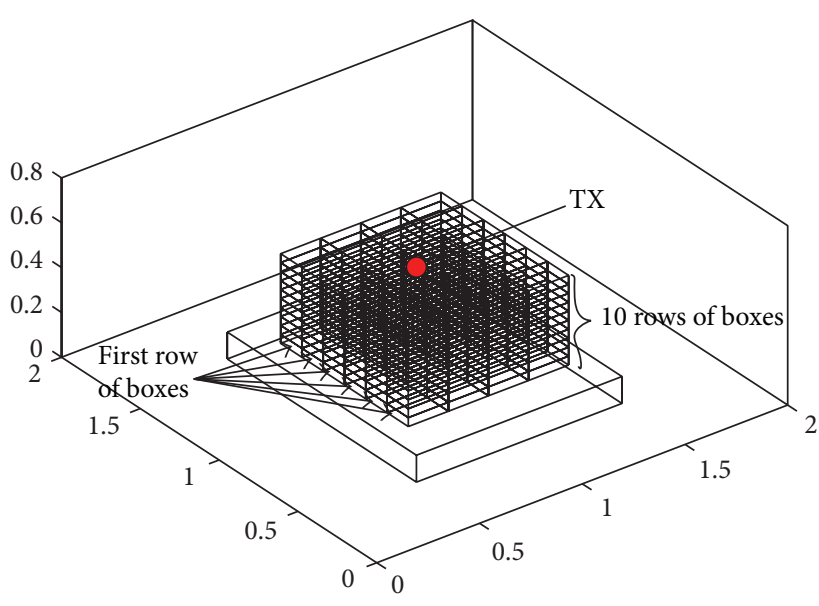

Figure 1: Details of the considered scenario for simulation.

TABLE 1: Different material properties.

\begin{tabular}{lcc}
\hline & $\varepsilon_{r}$ & $\sigma(\mathrm{S} / \mathrm{m})$ \\
\hline Polyvinyl chloride (PVC) & 4 & 0.12 \\
Polypropylene & 3 & 0.11 \\
Drinking water & 20 & 0.01 \\
Cellulose & 1.1 & 0.035 \\
\hline
\end{tabular}

TAble 2: Parameters in the ray launching simulation.

\begin{tabular}{lc}
\hline Frequency & $868 \mathrm{MHz}$ \\
Vertical plane angle resolution $\Delta \theta$ & $1^{\circ}$ \\
Horizontal plane angle resolution $\Delta \varphi$ & $1^{\circ}$ \\
Reflections & 6 \\
Transmitter power & $0 \mathrm{dBm}$ \\
Cuboids size & $4 \mathrm{~cm}$ \\
\hline
\end{tabular}

which is computationally demanding in the case of full wave simulation codes. Moreover, time domain results such as delay spread can be obtained, which can be employed for further uses, such as location applications.

In order to analyze the performance of the propagation channel in a typical scenario of traceability in a logistic company, a simplified pallet with boxes of different materials, which emulate different products, has been developed for the 3 D ray launching code. The pallet has $1000 \mathrm{~mm} \times 1200 \mathrm{~mm} \times$ $200 \mathrm{~mm}$ dimensions. Figure 1 shows a schematic view of the considered pallet for simulation with ten rows of 16 boxes each. The transmitter position is depicted with a red point just above the boxes, emulating the transmitter antenna of the RFID reader which is integrated in the pallet truck.

Several simulations have been performed to analyze the impact of different materials of the products on the pallet. The properties of the materials have been taken into account considering their dielectric constant and the loss tangent at the frequency range of operation of the system under analysis. These properties are shown in Table 1 . Table 2 shows the parameters used in the simulations in the $3 \mathrm{D}$ ray launching code. 


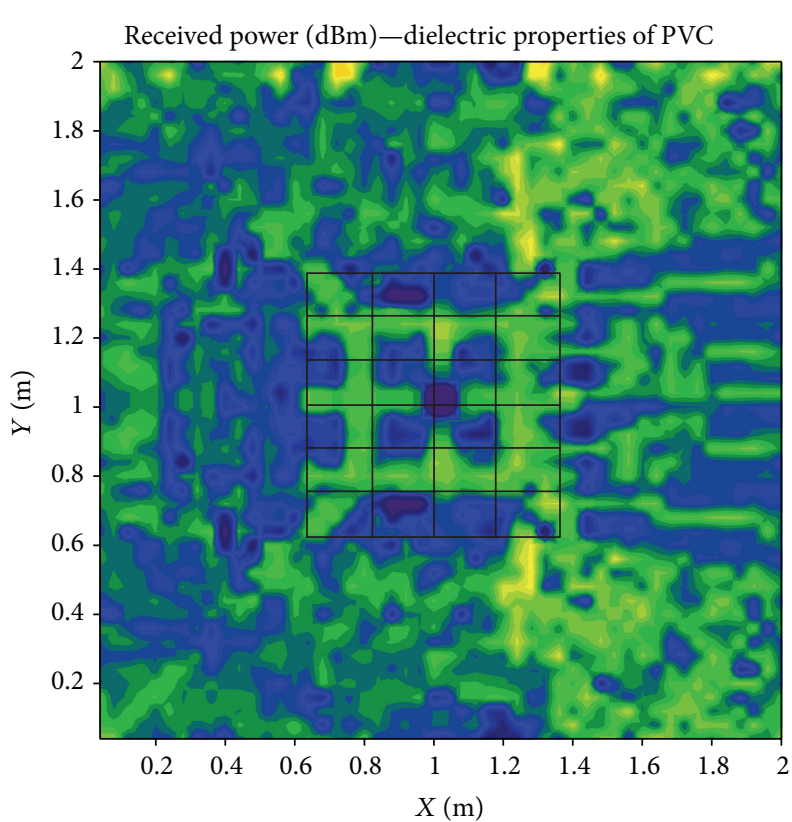

(a)

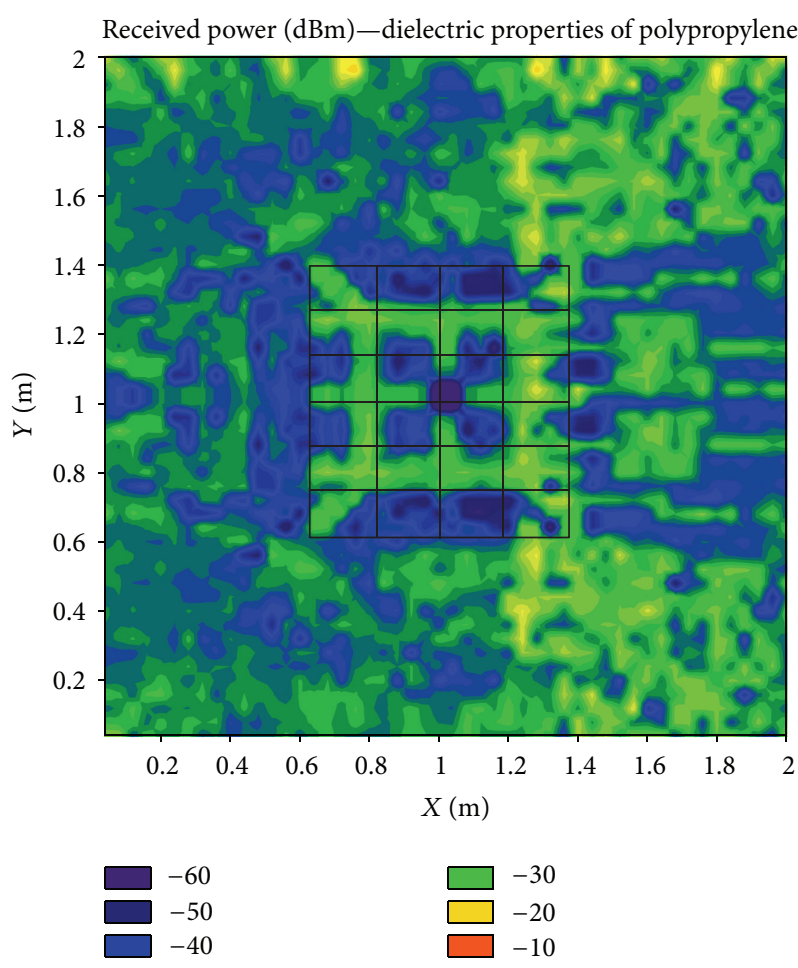

(c)

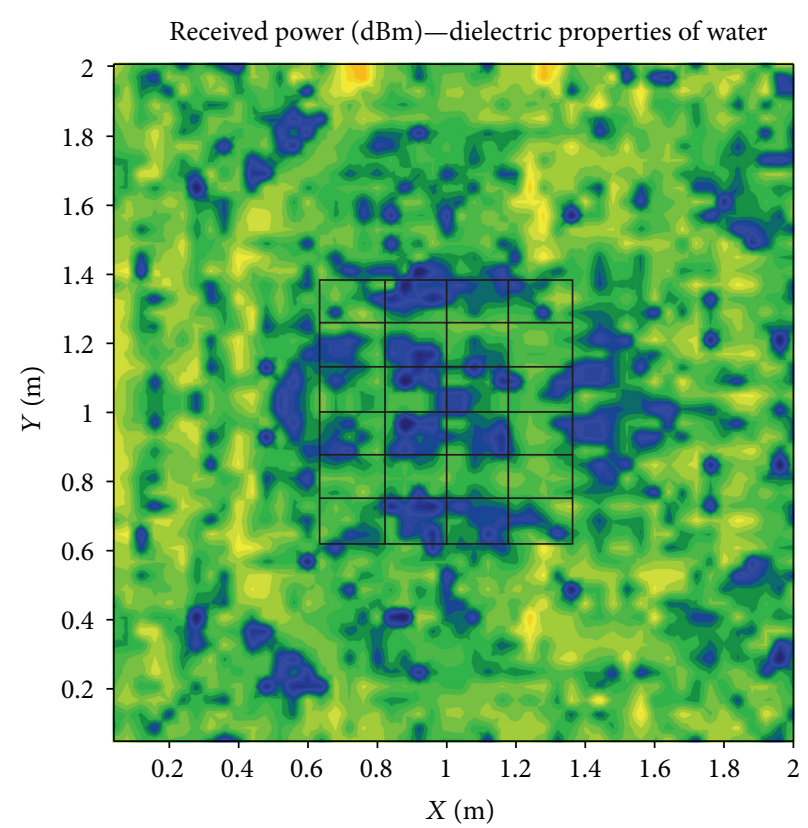

(b)
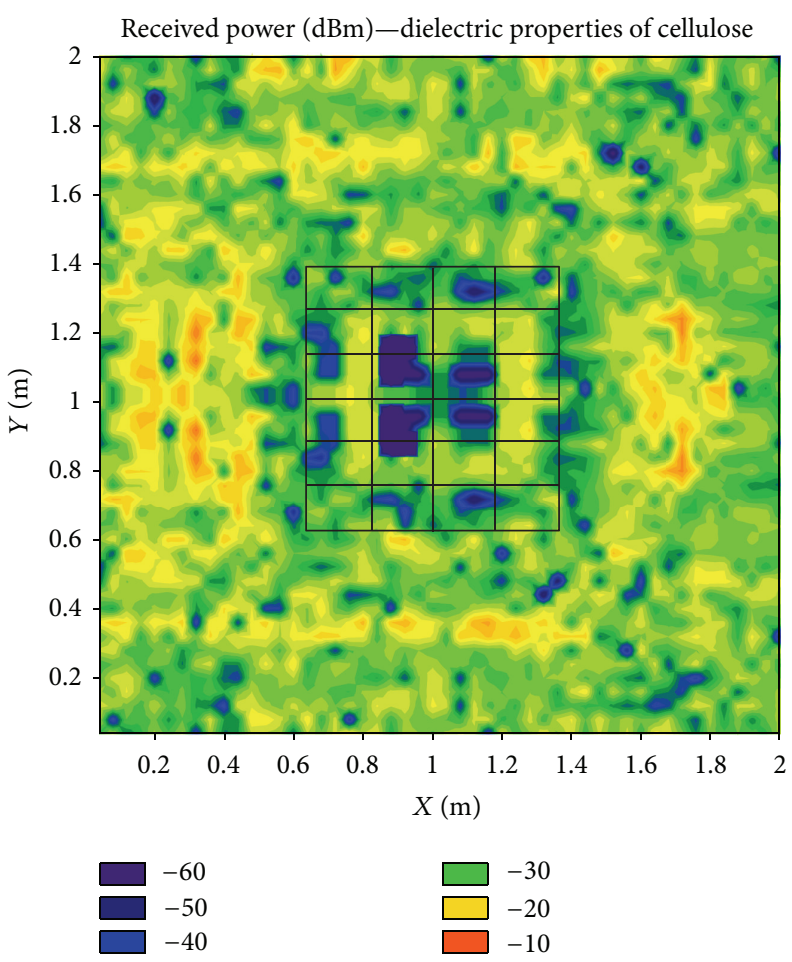

(d)

FIGURE 2: Received power $(\mathrm{dBm})$ for the first row of boxes in the pallet for different material products considered. (a) Polyvinyl chloride (PVC). (b) Drinking water. (c) Polypropylene. (d) Cellulose.

Figure 2 shows simulation results obtained by means of in-house 3D ray launching algorithm for the received power for the bidimensional plane for the first row of boxes in the pallet which is depicted in Figure 1. The received power is shown for different material products considered, emulating different pallets of the same product for traceability applications. It is observed that the material properties have a great impact on the radiowave propagation. For all cases, the received power in a tag which could be positioned in each box of the first row can be read because the received power is greater than the reader sensitivity. However, it is highly important to consider this radioplanning assessment because 


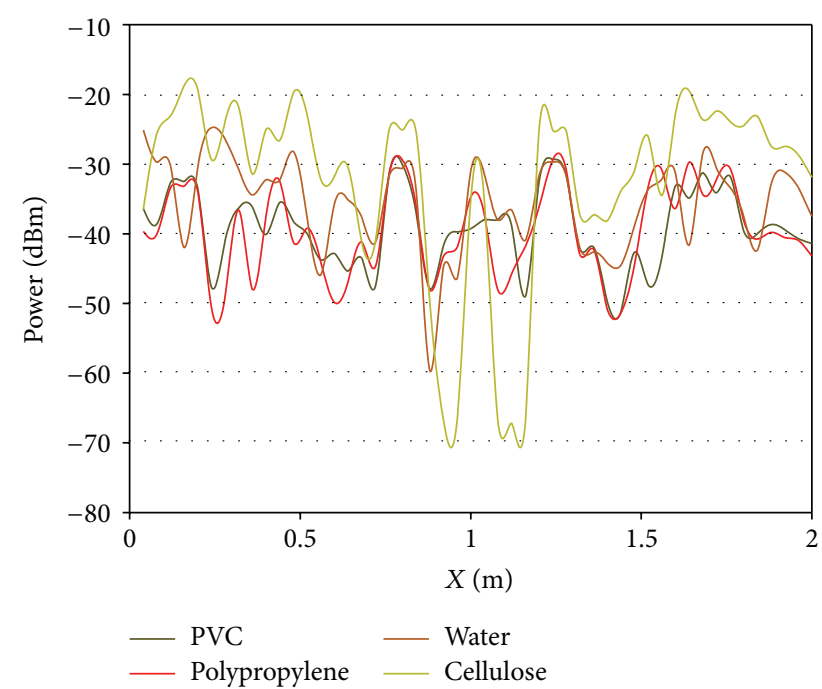

FIGURE 3: Radials of received power for different materials for the center of the first row of boxes.

the different dielectric properties of the materials could lead to unread tag cases and give errors in the traceability application.

The comparison among the different materials considered for the first row of boxes along the $x$-axis, for $Y=1.1 \mathrm{~m}$ is represented in Figure 3. It is observed a great variability between the different positions of the receiver. It is also shown that, for the cellulose material, the received power is slightly higher than for the other materials.

To validate previous predictions, measurements with a real pallet have been performed. Figure 4(a) shows the real pallet with the transmitter antenna just above the column of boxes. The tag antenna, Confidex Carrier a Class 1 Generation 2 passive UHF RFID Transceiver, was located inside the boxes randomly on the top of wrapped electronic circuit boards. The reader and antenna features are detailed in the next section. Figure 4(b) shows a schematic view of the considered pallet for simulation.

Figure 5 shows the comparison between simulation and measurements, exhibiting good agreement with a mean error of $0.67 \mathrm{~dB}$. The received power levels have been obtained by embedding a very short monopole connected to an Agilent Field Fox N9912A spectrum analyzer. The results indicate that the received power decreases with the distance to the transmitter due to the impact of the electronic circuit boards inside the boxes.

From the previous results, a clear dependence on the location of both reader and potential tag placement on the overall radiolink balance can be observed. Moreover, as the material properties of the elements which host the RFID tags as well as the intrinsic properties of the tags (i.e., tag radar crosssection and chip level mismatch) also have impact on the received power, it can be a limiting factor in the compliance with reader sensitivity. The simulation results for a single pallet with goods can be in principle extended to a large indoor scenario (warehouse, office building, etc.), and estimation of RF power levels for the complete indoor volume can be obtained. The estimation of the potential values of received power from the RFID system can be now employed in order to assess the reading range within the complete scenario under analysis and therefore aid in the deployment phase of the RFID readers within such scenario.

\section{Traceability System}

The proposed system requires active collaboration of manufacturers and assemblers of electronic devices tagging marketed devices individually through an UHF RFID tag. The use of standards such as EPC Gen $2[13,14]$ facilitates the future integration of the different manufacturers. Receiving docks in distribution warehouses must include RFID portals to detect and make an inventory of the contents of pallets shipped from the manufacturer. Likewise orders shipped from the warehouse to the final distributors are also registered using the same portals or handheld RFID readers for validating the correct reception.

According to radioelectrical analysis results shown in Section 3, a prototype of the system that uses exclusively handheld RFID reader has been implemented in order to validate the solution.

4.1. Hardware System and Communications. As mentioned previously, each delivery vehicle is fitted with a handheld reader to detect the SKU included in each pallet during load moments in the store and the store receipt. When transportation in the last stage of the distribution chain is carried out by third party carriers, the low cost of the portable reader (600€) allows end stores to have their own equipment to validate the receipt of orders.

To reduce the cost of this device and for the ease of use by different actors involved in the supply chain, the portable RFID reader lacks user interface and internet connection, requiring to be controlled from a mobile device as shown in Figure 6. In this sense it has been developed a mobile application for Android OS that allows interfacing the reader from a friendly interface that requires no training.

Once the user authenticates, the steps to perform the validation of a pallet are as follows.

(i) Identify the pallet: this task can be performed using the keyboard by entering the code of the pallet or, if the mobile device includes a camera, using the QR code included in the pallet identification label.

(ii) Enable reading: the reader device activates its receiving antenna and the device starts sending all labels of electronic devices that are identified.

(iii) Validate reading: finally, the identification of the pallet along with all the labels from electronic devices that have been identified is packaged in an XML file and sent, using $3 \mathrm{G}$ or WiFi, to the central server. Then, the central server processes the data and returns a message indicating a correct validation or reporting the tags and description of unidentified devices forming part of the pallet. 


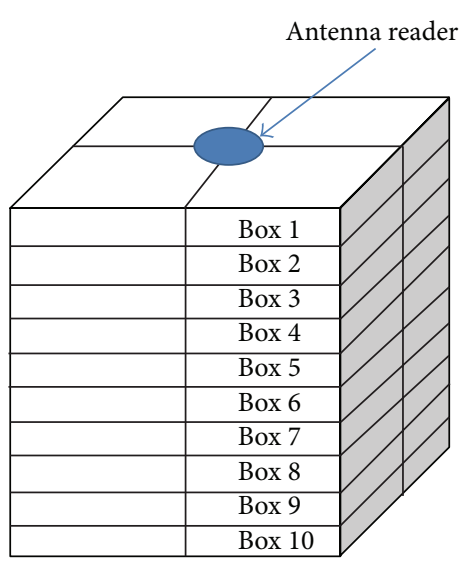

(a)

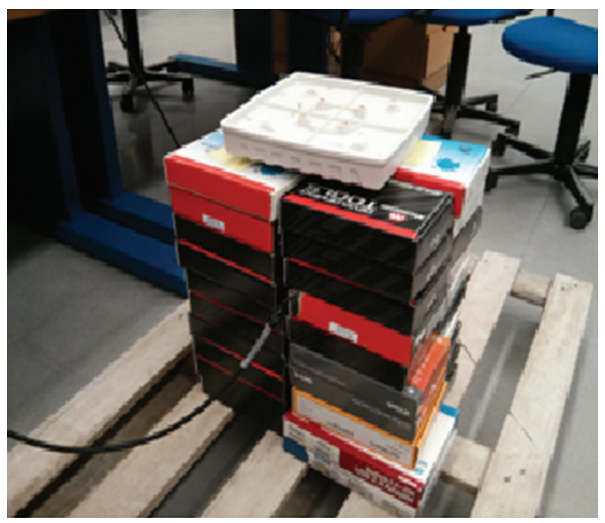

(b)

FIGURE 4: Radials of received power for different materials for the center of the first row of boxes.

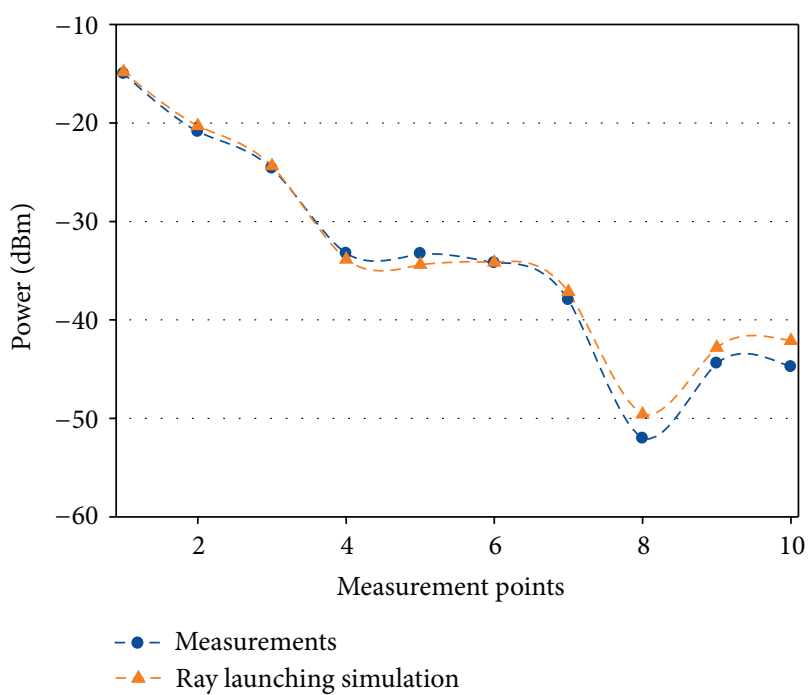

Figure 5: Comparison of simulation versus measurements for boxes 10 to 1 in the pallet.

The reader device prototype has been implemented trying to minimize the cost without compromising reliability in readings. It consists of three major subsystems.

(i) RFID reader: the prototype has been implemented using the $\mathrm{M} 5 \mathrm{e}$ compact embedded reader from ThingMagic. The reader provides support for EPCglobal Gen 2 (ISO 18000-6C) tag protocol with anticollision [15] and DRM capabilities. Despite its small size $(7 \times 10 \mathrm{~cm})$, it provides reading settable transmit level, adjustable from $10 \mathrm{dBm}$ to $23 \mathrm{dBm}$. In addition it supports 860 to $960 \mathrm{MHz}$ UHF RFID carrier frequency range to accommodate worldwide regulations and provides a maximum tag read rate of over 200 tags per second and maximum tag read distance of over 20 feet $(6 \mathrm{~m})$ using a $12 \mathrm{dBi}$ antenna.

(ii) UHF antenna: a commercial antenna has been used in the implementation of the prototype. Selected UHF

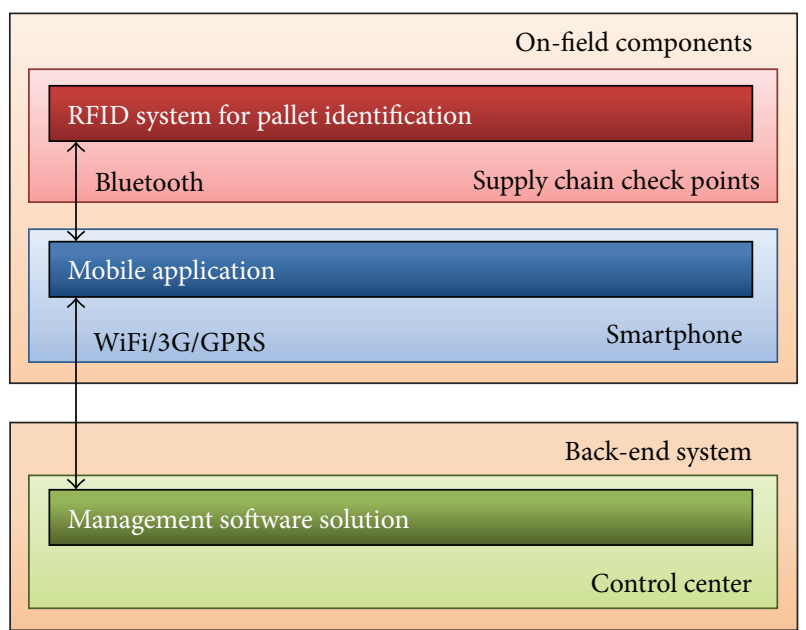

FIgURE 6: Interaction between the on-field and back-end System components of the proposed system.

antenna provides a gain of $6 \mathrm{dBi}$ and includes a 2meter cable to make easier the access to the pallet from different angles.

(iii) Embedded platform: an IGEP v2 industrial processor board manages the information provided by the reader and the communications with the mobile device. This card features an ARM Cortex A8 processor running at $1 \mathrm{GHz}$ and 512 megabytes of RAM and connects with the reader through a UART port to 115200 baud. The connection to the mobile device is carried out via Bluetooth. When the embedded platform receives the order to start reading from the mobile device, it sends the appropriate command to the RFID reader and stores all the identified labels in a data collection. Finally, when the phone sends the validate command, the reader is turned off, and data collection is sent to the mobile device. 


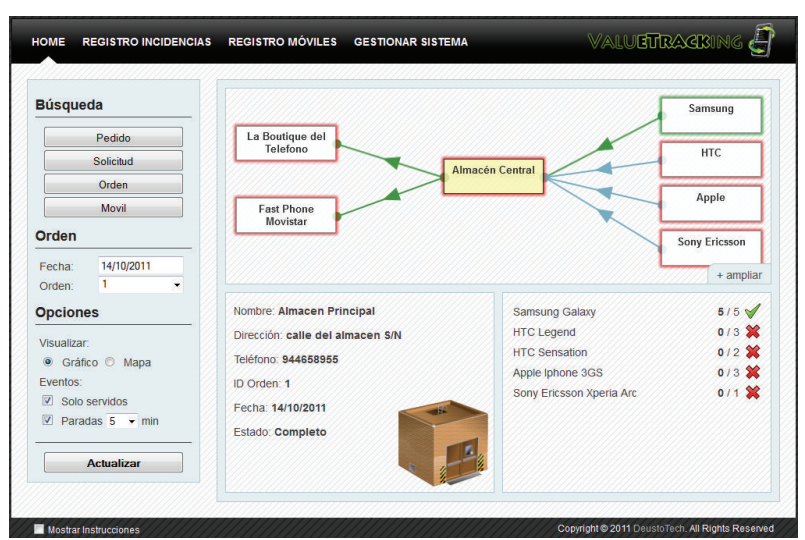

FIGURE 7: Management solution control panel web interface.

4.2. Logistics Software System. The developed software solution fulfills the mission of giving support to the proposed prototype architecture. To accomplish this, it is based on two independent but interrelated components: the mobile application and the management solution deployed on the central server.

The functionality offered by the mobile application has already been described in the previous section, performing mainly coordination and communication tasks, serving as an accessible entry point to the system, interacting and thereby integrating the functionality of the various components involved in the traceability process.

To achieve this, mobile application's software has the required configuration to allow interconnection via Bluetooth with reading devices in order to receive data from the performed RFID readings at each check point, as well as connectivity via web services with the centralized management solution.

In order to obtain the identification data for the shipment which is being processed, a QR reading service has been provided. As a result, approaching the $\mathrm{QR}$ code printed on the bill of delivery to the mobile application speeds up and largely automates the acquisition of relevant data (shipment code, participant identifier, priority, etc.) that would otherwise be introduced manually by an operator, with the waste of time and the increase in the probability of making mistakes which this would entail.

Finally, the mobile application presents a user-friendly interface through which the user is informed of the discrepancies in the actual and previous reading of pallets, indicating the potentially stolen items and generating and sending any incidences to the centralized management solution. The complete schematic of interaction between the readers and the management system is depicted in Figure 6.

This management solution is the key software component of the proposed solution as it complies with the necessity of maintaining, updating, and managing in an efficient way all the information received from the various participants in the supply chain, as well as providing the tools needed to facilitate an effective data sharing between heterogeneous devices and components.
The management software solution thus relates to a centralized application, deployed in a powerful back-end system, providing the system with data management and communications capabilities. This includes the development of a user control panel, Figure 7, whose main functionality is described as follows.

(i) Supply chain traceability: it provides both graphical and tabular information related to the current status of the shipments made by the system. It allows a complete pallet traceability, updating data, and informing the user when the readings and/or incidences from the supply chain check points are received. The application also offers search and filter functionality based on relevant criteria: order number, delivery date, dealer/shop identifier, model, and so forth.

(ii) Incidence management: incidents produced (resulting from a mismatch between subsequent readings) will be specifically managed by the system, registering them in the database and counting with various states (open, managed, closed) that enable its effective treatment by the administrator.

(iii) Electronic devices traceability: section rose to facilitate control and traceability of particular devices based on its specific brand, model, or EPC code. Historical device traceability, any incidences arising, and general shipping data will be also presented.

(iv) System management: specific section for the internal management of the system that enables CRUD (create, read, update, delete) operations on the participants of the supply chain as well as other internal management operations such as user registration and privilege management.

Besides the business capacities treated in the previous point, the architecture of the management software solution is divided into other functional layers following a modular structure that facilitates reuse, minimization of the coupling, and allowance for future functional enhancements in the prototype, Figure 8.

(i) Web services layer: It is developed by .NET technology, using WCF RIA services; this layer is responsible for coordinating requests made to the system from the different field elements. The use of this service layer promotes a safe and effective way for data communication between the components, managing at the same time system security by giving the necessary permissions.

(ii) Data persistence layer: Within this layer all information transferred between the different elements of the system was modeled. Microsoft SQL Server 2008 DBMS has been used in this context. The information related to each shipment is received and stored at the time the reading is performed at each check-point of the supply chain. In case any incidence was reported, this will be registered and stored in the database.

(iii) Communications layer: information relevant to the application is in the database; however, access to such 


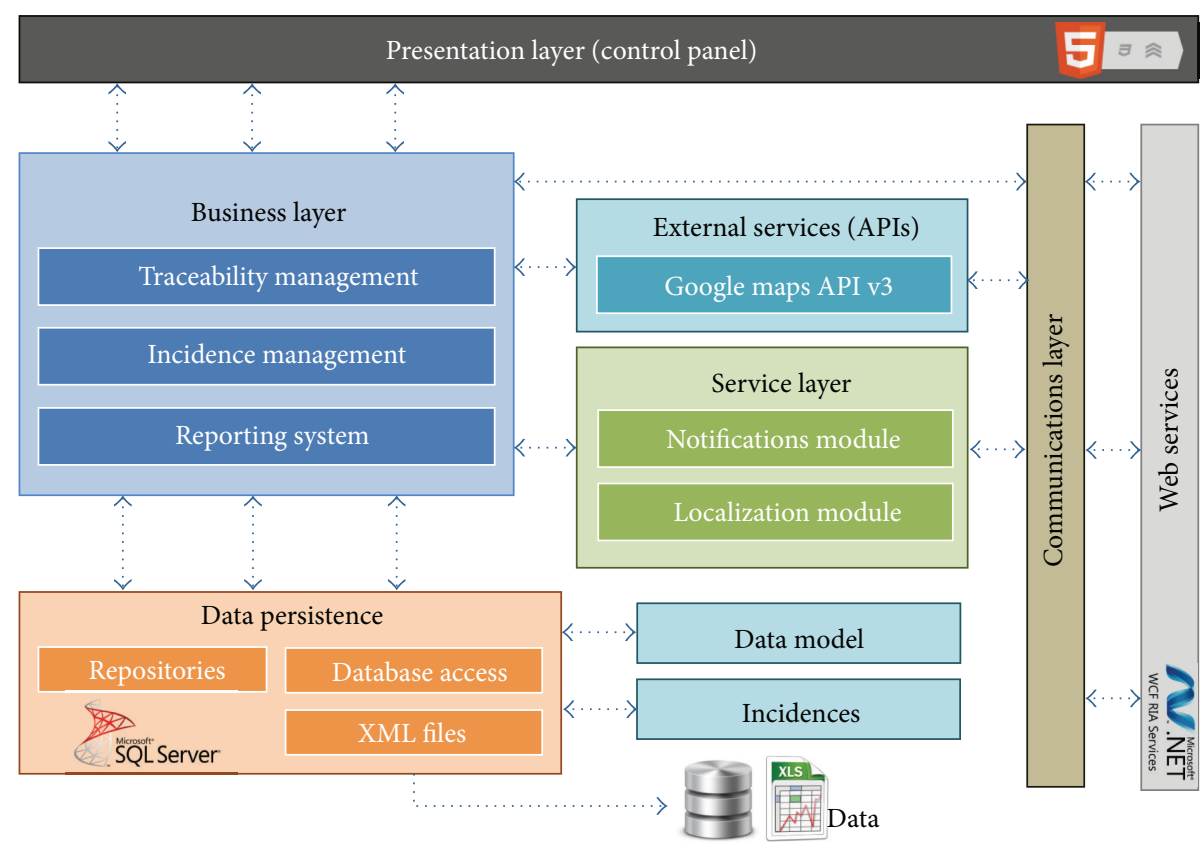

FIGURE 8: Architecture of the management software solution.

information is done through web services. This technical decision allows both data and application logic to be accessed from other devices, thereby ensuring the scalability and interoperability of the whole system. Security is also increased since data access does not occur directly but through the services, providing greater control over database queries.

The network will be controlled at all times under a firewall that prevents unauthorized access. The set of services developed allows full interoperability between the different components of the system, which is a major benefit in broadening the number of devices compatible with it and enabling their development in the future.

\section{Conclusions}

In this work, the use of RFID technology in order to improve logistic processes involving merchandise in pallets is presented. By the use of deterministic in-house $3 \mathrm{D}$ ray launching code, the influence on the election of material parameters as well as the arrangement of the different items within the pallet is obtained, which strongly conditions the performance of the system in terms of reading capacity. The complete system has been implemented in order to test the viability of its application in order to enhance logistical processes. The combination of physical layer analysis with a top layer system view can aid the planning as well as operational phase of this type of RFID system within a logistic chain.

\section{References}

[1] V. Kapsalis, C. Fidas, L. Hadellis, C. Karavasilis, M. Galetakis, and C. Katsenos, "A networking platform for real-time monitoring and rule-based control of transport fleets and transferred goods," in Proceedings of the 13th International IEEE Conference on Intelligent Transportation Systems (ITSC '10), pp. 295-300, Funchal, Portugal, September 2010.

[2] J. Cleland-Huang, R. Settimi, E. Romanova, R. Berenbach, and S. Clark, "Best practices for automated traceability," Computer, vol. 40, no. 6, pp. 27-35, 2007.

[3] D. Yue, X. Wu, and J. Bai, "RFID application framework for pharmaceutical supply chain," in Proceedings of IEEE International Conference on Service Operations and Logistics, and Informatics (IEEE/SOLI '08), pp. 1125-1130, Beijing, China, October 2008.

[4] T. Kelepouris, K. Pramatari, and G. Doukidis, "RFID-enabled traceability in the food supply chain," Industrial Management \& Data Systems, vol. 107, no. 2, pp. 183-200, 2007.

[5] G. Liu, W. Yu, and Y. Liu, "Resource management with RFID technology in automatic warehouse system," in Proceedings of IEEE/RSJ International Conference on Intelligent Robots and Systems (IROS '06), pp. 3706-3711, Beijing, China, October 2006.

[6] A. Moreno, I. Angulo, A. Perallos et al., "Intelligent van for the distribution of pharmaceutical drugs," Sensors, vol. 12, no. 5, pp. 6587-6609, 2012.

[7] S. F. Wamba and A. Wicks, "RFID deployment and use in the dairy value chain: applications, current issues and future research directions," in Proceedings of the IEEE Internationl Symposium on Technology and Society (ISTAS'10), pp. 172-179, Wollongong, Australia, June 2010.

[8] Indicod-Ecr: C.F. 80140330152, "Storia di successo-DHL, Mediamarket, Samsung, Sony," http://www.tendenzeonline.info/ news/dir/4/view/950/storia-di-successo-dhl-mediamarket-samsung-sony-95000.html.

[9] J. A. Nazábal, P. López Iturri, L. Azpilicueta, F. Falcone, and C. Fernández-Valdivielso, "Analysis of IEEE 802.15.4 compliant wireless devices for heterogeneous indoor home automation environments," International Journal of Antennas and Propagation, vol. 2012, Article ID 176383, 14 pages, 2012. 
[10] L. Azpilicueta, F. Falcone, J. J. Astráin et al., "Measurement and modeling of a UHF-RFID system in a metallic closed vehicle," Microwave and Optical Technology Letters, vol. 54, no. 9, pp. 2126-2130, 2012.

[11] E. Aguirre, J. Arpón, L. Azpilicueta, S. de Miguel, V. Ramos, and F. Falcone, "Evaluation of electromagnetic dosimetry of wireless systems in complex indoor scenarios with human body interaction," Progress in Electromagnetics Research B, vol. 43, pp. 189209, 2012.

[12] P. L. Iturri, J. A. Nazábal, L. Azpilicueta et al., "Impact of high power interference sources in planning and deployment of wireless sensor networks and devices in the $2.4 \mathrm{GHz}$ frequency band in heterogeneous environments," Sensors, vol. 12, no. 11, pp. 15489-15708, 2012.

[13] EPCglobal, EPC Radio-Frequency Identity Protocols Class-1 Generation-2 UHF RFID Protocol for Communications at 860 $\mathrm{MHz}-960 \mathrm{MHz}$ Version 1.0.9.

[14] K. Chiew, Y. Li, T. Li, and R. H. Deng, "On false authentications for C1G2 passive RFID tags," in Radio Frequency Identification System Security, pp. 50-65.

[15] H. Landaluce, A. Perallos, I. J. G. Zuazola, and Y. I. Angulo, "Performance analysis of the Slot Counter algorithm in comparison with the real performance of a commercial RFID reader supporting the EPC class 1 generation 2 protocol," in Proceedings of the 20th International Conference on Software, Telecommunications and Computer Networks (SoftCOM '12), pp. 1-5, Split, Croacia, 2012. 

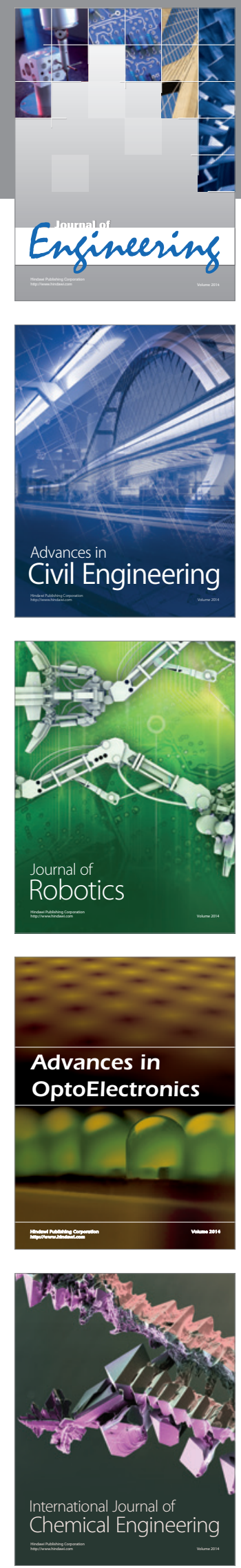

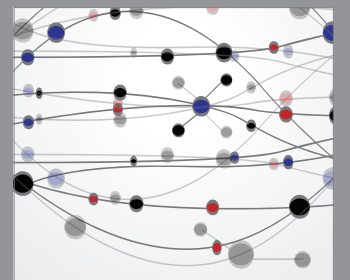

The Scientific World Journal
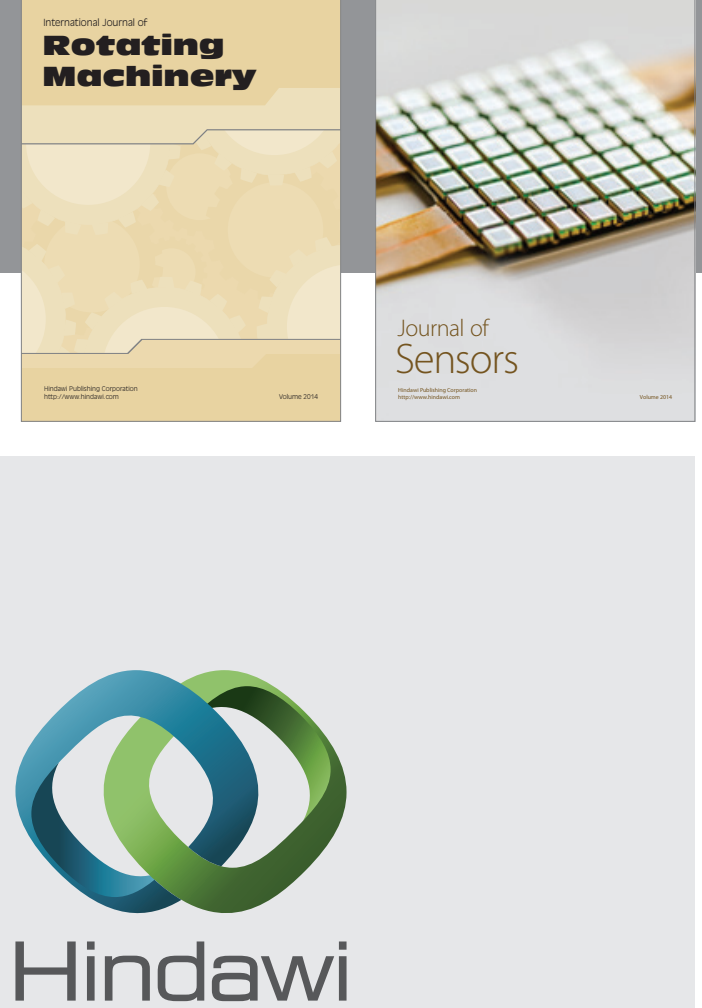

Submit your manuscripts at http://www.hindawi.com
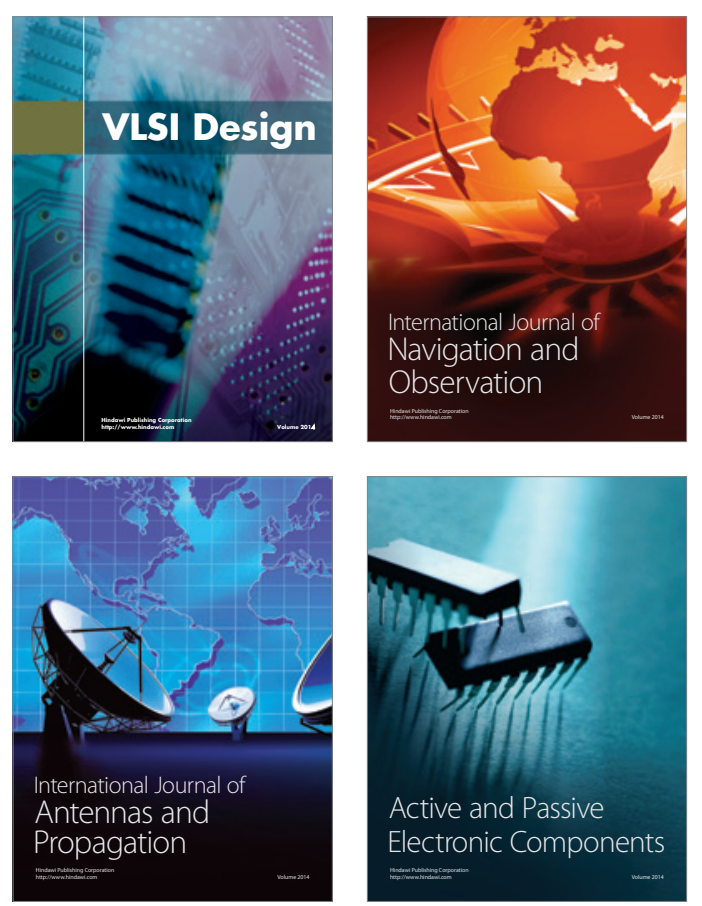
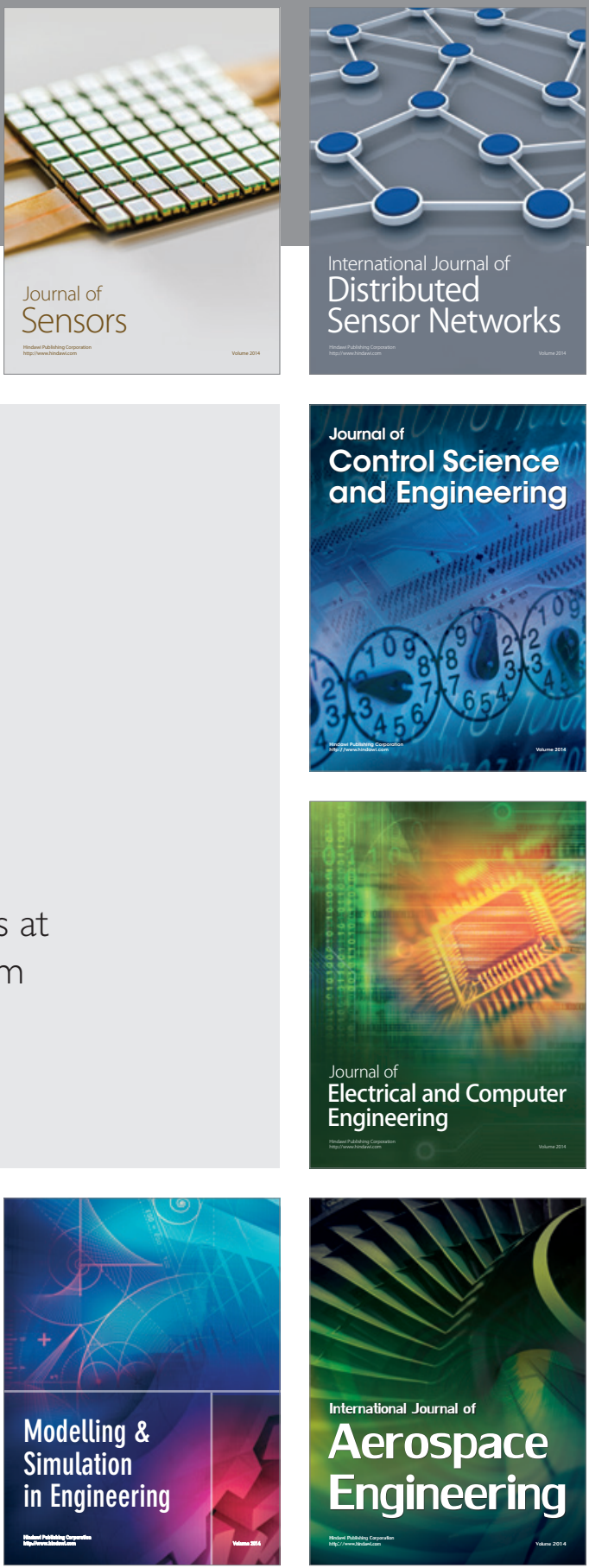

Journal of

Control Science

and Engineering
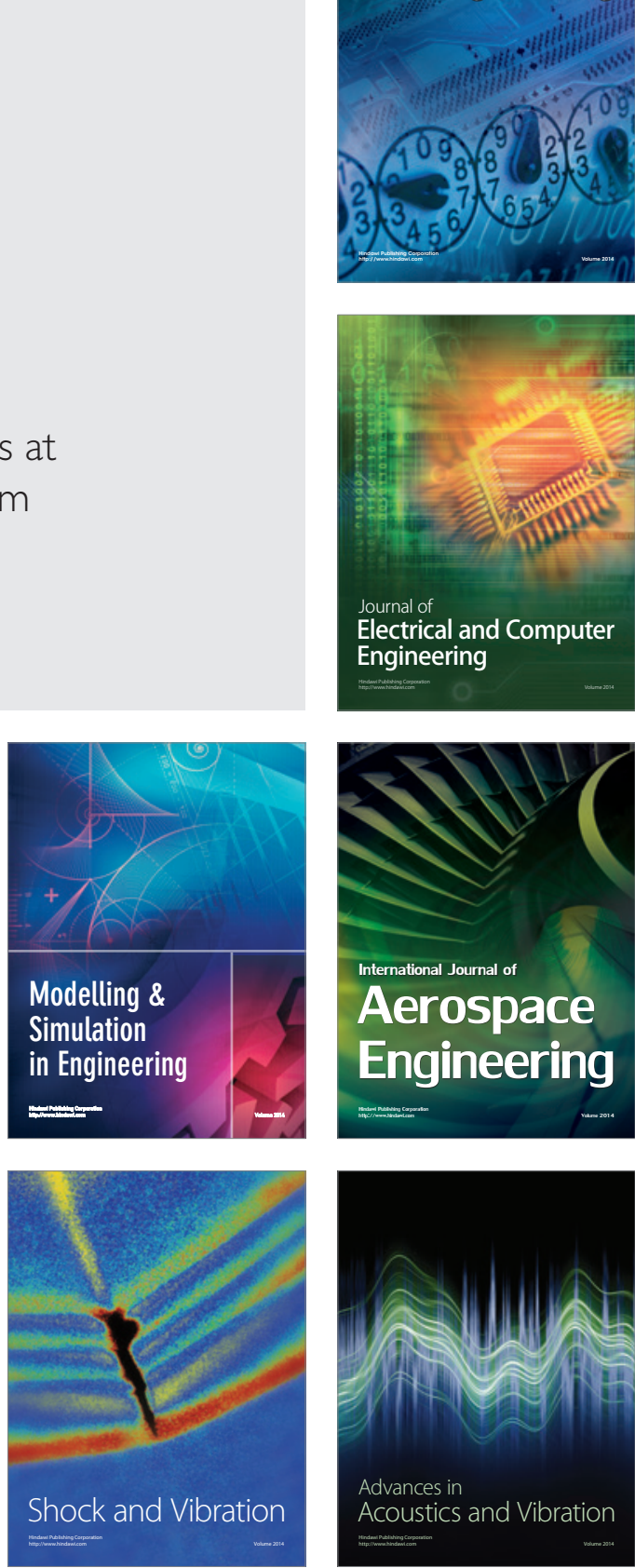